\title{
Resúmenes de la Literatura Actual
}

\section{MEDIDA DEL AREA BAJO LAS CONTRACCIONES COMO EXPRESION DE LA ACTIVIDAD UTERINA}

\author{
Dres.: Edgard Cobo y Carlos A. Quintero
}

La actividad uterina se ha definido como el resultado de multiplicar la intensidad por la frecuencia de las contracciones, y se expresa en Unidades Montevideo (1). Sin embargo, una contracción uterina está compuesta por otros elementos, además de la intensidad y la frecuencia, que no están incluídos en esta definición. Estos elementos son: la forma, la duración y el tono de las contracciones.

La medida del área, representaría una posibilidad de integrar en un solo valor todos estos elementos, propios de las contracciones del útero humano grávido.

El objetivo de este trabajo es describir nuestros resultados, tendientes a estudiar esta última posibilidad de medida.

\section{Material y Métodos}

Hemos medido el área de 5.821 contracciones uterinas, en 1.170 períodos de 10 minutos, con el objeto de comparar los valores así obtenidos, con los valores de "actividad uterina" correspondientes a esos mismos períodos. 611 períodos corresponden a partos normales; 287 casos de Abruptio Placentae, (A.P.) Tipo I y 272 casos del Tipo II (2).

El área se obtuvo por planimetría y se expresó en $\mathrm{mm} 2$. por 10 minutos.
Se estudió el índice de correlación entre área y "actividad uterina" para cada uno de estos grupos y además se estudió la distribución de frecuencias de cada grupo, tanto para el área, como para la "actividad uterina".

\section{Resultados}

El índice de correlación mostró valores positivos para los tres grupos, siendo de 0.66 ( 0 a \pm 0.92 al nivel de probabilidad de 0.95 ); de 0.65 (0.38 a \pm 0.96 ) para el grupo A.P. Tipo I y de 0.43 (- 0.88 a +0.95$)$ para el grupo A.P. Tipo II.

La distribución de frecuencias fue bastante similar en los tres grupos, cuando la medida fue la actividad uterina expresada en Unidades Montevideo. En cambio, esta distribución fue diferente para todos los grupos, cuando la medida fue el área bajo las contracciones.

\section{Comentario}

Nuestros resultados indican una correlación aceptable (aunque con una dispersión alta) en los casos de parto normal.

Esta correlación sigue siendo buena (aunque con una dispersión mayor) en los casos de Abruptio Placentae, Tipo I, en los cuales hemos descrito una contractilidad con mu- 
chas semejanzas a la contractilidad normal. En los casos del Tipo II, en los cuales hay alteraciones de todos los elementos de las contracciones uterinas, la correlación no existe.

La distribución de frecuencias demuestra que no hay una discriminación clara entre los 3 grupos, cuando se mide convencionalmente la actividad uterina a pesar de existir variaciones apreciables en el patrón de actividad contráctil.

En cambio, utilizando el área, como expresión de la actividad del útero, la discriminación es clara.
En consecuencia, concluímos que la medida del área bajo las contracciones uterinas, es un método que permite discriminar cuantitativamente las variaciones normales y las alteraciones patológicas de la contracción uterina, de una manera más integral que otras medidas convencionales.

\section{BIBLIOGRAFIA}

1 ALVAREZ, H., and CALDEYRO-BARCIA, R. : Surg. Gynec. \& Obst. 91: 1, 1950.

2 COBO, E., QUINTERO, C. A., STRADA, G. and ZAMBRANA, M. A. Am. J. \& Gyn. 93: $1151 ; 1965$.

\section{INHIBICION DE LA FUNCION NEUROHIPOFISIARIA EN LA LACTANCIA HUMANA}

\section{Dr. Edgard Cobo}

\section{Resumen}

En publicaciones previas (1.2) establecimos que la neurohipófisis humana liberaba independientemente oxiltocina u hormona antidiurética, dependiendo del estímulo empleado. Con el objeto de explorar más a fondo estos hallazgos experimentales, diseñamos un estudio de la función neurohipofisiaria, usando sistemas inhibidores, como la sobrecarga de agua y el alcohol, simultáneamente con estímulos de la secreción de oxitocina, como: la succión del niño.

\section{Material y Métodos}

Se estudiaron 73 pacientes normales, entre el $2^{\circ}$ y el $8{ }^{\circ}$ día de la lactancia. En todas se establecieron valores controles. En 34, se administró agua (Dextrosa al $5 \%$ en A.D.) i.v. en cantidades de $20 \mathrm{cc}$. por $\mathrm{Kg}$. de peso. En 31, se administró alcohol, hasta lograr concentraciones sanguíneas entre 50 y $390 \mathrm{mg} . \%$.

Las actividades antidiurética y eyecto-láctea se estudiaron con métodos descritos previamente $(1,2)$.

Se realizaron curvas efecto-dosis, con oxitocina exógena, para estar seguros de que la inhibición obtenida no afectaba la glándula mamaria y era en consecuencia central, o neurohipofisiaria, y no periférica, o mamaria.

\section{Resultados}

Los resultados obtenidos demuestran: 1. Que cuando se administra agua 0 alcohol hasta obtener concentraciones bajas (50-100 mg.\%). la actividad antidiurética espontánea se inhibió, cuando al mismo tiempo la actividad eyecto-láctea espontánea no 
mostró ninguna inhibición, y más aún, mostró un aumento marcado en 3 experimentos. Dicho en otras palabras, que existe una inhibición diferencial de la neurohipófisis.

2. Esta misma afirmación es válida para el efecto de la succión del bebé, realizada durante la administración de los agentes inhibidores.

3. Las concentraciones sanguíneas de alcohol, altas (200-400 mg.\%), producen una inhibición marcada de ambas actividades.

\section{Comentario}

Nuestros resultados demuestran que la inhibición de la glándula neurohipofisiaria humana, es diferencial en ciertas condiciones experimentales. Este hecho refuerza nuestro postulado previo, que sugiere la existencia de vías neurales aferentes y/o mecanismos de liberación, independientes para cada una de las hormonas neurohipofiisarias, oxitocina y hormona antidiurética.

\section{BIBLIOGRAFIA}

1 COBO, E., GAITAN, E., MIZRACHI, M. and STRADA, G. Neurohypophyseal Hormone release in the human. Am. J. Obst. \& Gynec. 91: $905 ; 1965$.

2 COBO, E., DE BERNAL, M., GAITAN, E., and QUINTERO, C. A. Neurohypophyseal Hormone release in the Human. II Experimental study during Lactation. Am. J. Obst. \& Gynec. 97: $519 ; 1967$.

\section{ASPIRACION UTERINA EN EL TRATAMIENTO DEL ABORTO INCOMPLETO}

(Eaton, C. J. y Doll, K. L.; Universidad de Michigan, Centro Médico). Surg. Gynec. Obstet. 129: 588-589, 1969.

De 150 pacientes con diagnóstico de aborto incompleto entre la sexta y veinticincoava semana de embarazo, a todas se trató con aspiración uterina. De éstas, 70 pacientes $(40 \%)$ sufrieron aborto prematuro hasta de 12 semanas, y 80 pacientes (53\%) tuvieron aborto tardío de más de 12 semanas; 33 pacientes (22\%) eran consideradas sépticas. El curetaje (raspado) convencional se utilizó como procedimiento de control después de la aspiración uterina en todas las pacientes de esta serie.

No se presentó residuo significativo de tejidos en el control por raspado en ninguna de las pacientes con aborto prematuro incompleto. De las 80 pacientes con aborto incompleto tardío, $53(66 \%)$ fueron evacuadas en forma incompleta. Sin embargo, todas con excepción de 2 pacientes, presentaron reducción considerable en el tamaño del útero, produciendo un útero más seguro para completar en él el procedimiento por medio de una cureta cortante. La evacuación duró menos de 2 minutos en las pacientes con aborto prematuro y un promedio de 3 minutos en las pacientes con aborto tardío. La pérdida de sangre fué inferior a los $100 \mathrm{ml}$. en $76 \%$. Se pudo utilizar una cánula de $10 \mathrm{~mm}$. sin producir mayor dilatación en la mayoría de las pacientes. La paciente sufre muy poca o ninguna incomodidad por causa de la aspiración.

No se presentó morbilidad atribuíble a la aspiración uterina, ni perfo- 
raciones uterinas, y ninguna paciente necesitó de una segunda operación por motivos de residuo de tejidos.

La aspiración uterina puede ser tan útil para el tratamiento de abor- to incompleto como para interrupción terapéutica. Al evitar el curetaje vigoroso cortante en pacientes con abortos sépticos es menos probable que ocurra la diseminación de la infección.

\section{EXAMEN DEL LIQUIDO AMNIOTICO COMO AYUDA PARA DETERMINAR LA EDAD DE LA GESTACION}

(Droegemueller, W., Jackson, C. Makowski, E. L. y Battaglia, F. C. Centro Médico de la Universidad de Colorado). Amer. J. Obstet. Gynec. 104: 424-428, 1969.

Las concentraciones de creatinina y bilirrubina en el líquido amniótico y el porcentaje de células sebáceas fetales se determinó simultáneamente en 65 pacientes normales de obstetricia, quienes tenían una historia segura de la menstruación. De las tres; la concentración de creatinina en el líquido amniótico se encontró como la más segura para determinar la edad de la gestación al ser comparada con la edad de la gestación calculada sobre la historia de menstruación y confirmada por el examen físico y neurológico del recién nacido.
Después de 36 semanas de gestación, la concentración de creatinina era de $1.8 \mathrm{mg} . \%$ o superior. $\mathrm{Ni}$ la concentración de bilirrubina ni el porcentaje de células fetales indicaron una correlación apropiada con la edad de la gestación que tuviera valor clínico importante. Debido a que el líquido amniótico es de fácil accesibilidad y a que la concentración de creatinina puede ser medida fácilmente, esta técnica ofrece a los obstetras un criterio objetivo adiciona! para determinar la edad de la gestación antes del parto.

\section{INSEGURIDAD DE LA EVALUACION SENCILLA DEL PH EN LA SANGRE DEL CUERO CABELLUDO DEL FETO}

Lumley, J., et al. (Queen Victoria Hospital, Melbourne, Australia); J. Lab. Clin. Med. $77: 535-542,1971$.

Dos problemas condujeron a esta serie de experimentos: (1) el descubrimiento de una variedad de valores para el $\mathrm{pH}$ y los gases sanguíneos al efectuarse diferentes mediciones en alícuotas de una misma muestra de sangre del cuero cabelludo del feto, y (2) el descubrimiento de que más o menos un $10 \%$ de los pacientes diagnosticados como acidóticos $(\mathrm{pH}$ 7.20) tenían pH normal cuando más tarde se recolectó la sangre del feto. Estos dos problemas, junto con el descubrimiento universal de que el 
$\mathrm{pH}$ del feto no constituye una guía totalmente segura para determinar el estado del feto, dio origen al interrogante sobre errores en la medición del $\mathrm{pH}$. Sin embargo, la medición del $\mathrm{pH}, \mathrm{PCO} 2$ y $\mathrm{PO} 2$ con una precisión satisfactoria se obtuvo al analizar la sangre recolectada en una misma pipeta por medio de punción con aguja del cordón umbilical. El hecho de que la sangre fetal en el cordón no muestre tan amplia variación, muestra que no constituye una propiedad general del feto en oposición a la sangre adulta.

Estos experimentos, destinados a evaluar los cambios en la composición de la sangre del feto, asociados con el método de recolección de la misma en el cuero cabelludo, indicaron que algunas de las variaciones continúan siendo inexplicables. Existe gran posibilidad de que reflejen una composición variable de la sangre del cuero cabelludo del feto.

\section{PROTEINA C REACTIVA DEL SUERO EN EL EMBARAZO, EN EL PARTO Y EN EL PUERPERIO}

Jones, W. R. (Universidad de Sydney, Sydney, N. S. W., Australia). Aust. New Zeal. J. Obstet. Gynaec. 10: 218, 221, 1970.

La proteína C-reactiva del suero $(P-C R)$ se estudió en 202 mujeres embarazadas, parturientas y puerperales. La incidencia de pruebas positivas progresivamente aumentó a través de las diferentes etapas positivas terminando en una tasa del $97 \%$ después del primer día post-parto. Se notó un aumento brusco en la incidencia cuando los trabajos del parto se prolongaron por más de 12 horas, sugiriendo una asociación de la aparición de la P-CR con los cambios dinámicos del tejido a un nivel úteroplacentario.
Este problema representa una fase aguda de un proceso más sutil presente en las 29 pacientes que tuvieron una prueba positiva de P-CR en el tercer trimestre de embarazo. La aparición de esta proteína en el suero de mujeres embarazadas puede indicar cambios en el tejido intrauterino basados en variaciones biológicas (inmunológicas?) menores causadas por la relación feto-materna. La P-CR se encontró en 2 de los 70 cordones examinados, lo cual confirma su incapacidad para atravesar la placenta.

\section{EVALUACION DEL POSIBLE USO DE PROSTAGLANDINA E-1, E-2 y F-2, PARA INDUCIR EL PARTO}

Roth-Brandel. U. y Adams, M. (Karolinska Sjukhuset, Estocolmo, Suecia). Acta Obstet. Gynaec. Scand. 49 (Sup. 5) : 9-17, 1970.

Se administraron prostaglandinas por infusión intravenosa y se registró contracción uterina por el método intra-amniótico (Caldeyro Barcia et. al.). En las primeras series se aplicaron las prostaglandinas por la tasa de infusión aumentada gradualmente, manteniendo el nivel de cada do- 
sis durante 60 minutos. La prostaglandina E1 (PGE1) se administró a 8 pacientes y la prostaglandina $F 2 a$ ( $P$ GF2a) a otros 8 pacientes. Los resultados revelaron que una tasa baja de infusión (0.4 ug. PGE, y 3.0 ug. PG $\mathrm{F} 2 \mathrm{a} /$ minuto) aumentó primordialmente la frecuencia de las contracciones, mientras que el aumento de la intensidad solo fué aparente a niveles de dosis más elevadas. Con las tasas de infusión de 1.0 a $1.2 \mathrm{ug}$. PGE, 09.0 a 13.5 ug. PGF2a/minuto, la frecuencia de las contracciones excedió de 5/10 minutos en varias pacientes.

Para el estudio de la prostaglandina E2 (PGE2), que tiene aproximadamente la misma potencia de la PGE1 en lo que se refiere al útero humano, se administró a 13 pacientes embarazadas como infusión intravenosa continua a una tasa constante de 0.7 ug. minuto por un período de máximo 10 horas. En 7 de estas pacientes se presentó parto normal como resultado de la infusión, dentro de las 4 a 21 horas. Los signos de hiper-actividad uterina en una de las pacientes ocurrió después de 8 horas de estímulo y se realizó una cesárea debido a bradicardia en el feto. En las 5 pacientes restantes las contracciones desaparecieron después de la infusión.

El análisis de los valores medios de intensidad y frecuencia de las contracciones en 8 controles de la serie, donde las membranas permanecieron intactas durante el período de infusión, reveló que la intensidad aumentó gradualmente a $30-35 \mathrm{~mm}$. Hg y que la frecuencia alcanzó un valor medio de 4 a 5 contracciones cada 10 minutos. Como resultado, la actividad uterina permaneció comparativamente baja a pesar de la alta frecuencia. Además, tuvieron lugar episodios complejos de contracción no coordinada. La conclusión es que la prostaglandina puede ser usada para inducir los trabajos a término, pero que las sensibilidades individuales varían dentro de una gran gama y que hasta ahora no existen pruebas de que las prostaglandinas sean superiores a la oxitocina para este fin.

\section{EMBARAZO NO DESEADO}

(Sloane, R. B., Universidad de Temple, Centro de Servicios de Salud).

New Eng. J. Med. 280: 1206-1212, 1969.

No existen indicaciones psiquiátricas específicas para el aborto terapéutico. El riesgo de precipitación o exasperación en una psicosis existente es pequeño e impredecible y rara vez se presenta el suicidio. Las razones humanitarias con frecuencia determinan la decisión, aunque puedan ser disfrazadas con etiquetas psiquiátricas. Es poca la presencia de enfermedades psiquiátricas después de un aborto terapéutico, que puedan rela- cionarse con el procedimiento. La mujer que busca el aborto, por cualquier razón, puede resultar en una madre poco satisfactoria si se le forza a continuar su embarazo hasta el parto. Los síntomas emocionales pueden desaparecer más rápidamente si se cumple con sus deseos.

Cualquier cambio en la ley de abortos deberá incluir una cláusula so- 
cial; por ejemplo, la promulgada en Inglaterra. Esta reconoce el riesgo de un embarazo no deseado para la salud física y mental de los niños existentes y permite una consideración del temor de hacinamiento y tensión de la madre. Tal cambio facilitaría una evaluación más simple del aborto como una terapéutica en lugar de como un problema médico-social.

\title{
DIAGNOSTICO MATERNO POR IRRADIACION DE RAYOS $X$ ANTES DE LA CONCEPCION Y FRECUENCIA DEL MONGOLISMO EN NIÑOS NACIDOS POSTERIORMENTE
}

\author{
Stevenson, A. C., Mason, R. y Eduards, K. D. (Consejo de Investigación Médica, Unidad de \\ Genética de Población, Oxford, Inglaterra). Lancet, 2: 1335-1337, 1970.
}

Se ha sugerido que los procedimientos para diagnóstico por rayos $X$ antes de la concepción aumentan la frecuencia del mongolismo en los niños nacidos posteriormente. Para examinar esta teoría se realizaron investigaciones en 3.267 mujeres entre los 15 y los 40 años que habían sido sometidas a salpingogramas $u$ otros exámenes específicos de diagnóstico que implicaron exposición de los ovarios; 645 de estas pacientes suministraron información sobre niños concebidos después de los procedimientos de irradiación.

La dosis media gonadial evaluada antes de la concepción de los niños fue de 900 milirads en Oxford, mientras que en Toronto, Canadá, fue de 1.400 milirads. Los exámenes especificados para el grupo de mujeres en Oxford fueron salpingograma, posición del feto, pelvimetría lateral, radiografía del abdomen, urografía de excreción, pielografía retrógrada, uniones sacroilíacas, columna vertebral (lumbar), alimento de barium y su control, y enema de barium. Las
645 madres tuvieron 1.052 niños después de la irradiación —el intervalo promedio entre la última exposición y la concepción fue de aproximadamente 31 meses. De estos niños únicamente 3 presentaron el síndrome de Drown; todos tres tuvieron trisomía 21. Las 3 madres fueron sometidas a urografía de excreción pero a ningún otro examen radiográfico antes de la concepción de sus hijos. Todas tenían entre 35 y 39 años. En la serie de Oxford hubo 2 niños que presentaron trazos recesivos de gene y 16 que presentaron anormalidades de tipos comunes. Entre los 1.052 nacimientos hubo 14 niños que nacieron muertos.

La posibilidad de una divergencia seria en este tipo de investigaciones no puede ser pasada por alto. Sin embargo, este estudio no confirma el punto de vista de que los procedimientos radiológicos aplicados antes de la concepción aumenten la frecuencia del mongolismo en niños que nacen posteriormente. 


\section{NIVELES DE GLUCOSA SANGUINEA Y TOLERANCIA DE GLUCOSA EN MUJERES PRE-DIABETICAS Y SUB-CLINICAMENTE DIABETICAS CON UNA DOSIS BAJA DE CONTRACEPTIVO DE PROGESTOGENO}

Goldman, J. A. y Eckerling, B. (Geilinson Medical Center, Tel Aviv, Israel).

Israel J. Med. Sci. 6: 703-707, 1970.

Los estudios preliminares han confirmado las alteraciones en glucosa sanguínea y la tolerancia de glucosa, así como la insulina en el plasma en mujeres sanas, en mujeres pre-diabéticas, sub-clínicamente diabéticas y francamente diabéticas, en combinación con contraceptivos orales de tipo de secuencia. Además, se encontró que la progesterona no tiene efecto alguno sobre el metabolismo de carbohidratos de la mujer, en comparación con el estrógeno. Las actuales investigaciones se realizaron con el fin de determinar el efecto de un contraceptivo oral de tipo de la progesterona (ćliacetato etinodinodiol) sobre la glucosa sanguínea y la tolerancia intravenosa de glucosa. Las tasas de glucosa sanguínea fija y de desaparición de la glucosa sanguínea se midieron con ayuda de una prueba de tolerancia intravenosa de glucosa (GTT) en mujeres con presumible pre-diabetis y sub-clínicamente diabéticas; todas estaban siendo tratadas con una dosis oral baja de agente contraceptivo de progesterona.
Antes de iniciar el contraceptivo oral y hasta 3 meses después de la terapia, se aplicó a cada paciente un GTT intravenoso. La glucosa sanguínea fija se volvió a medir 3 meses después de terminada la terapia. La glucosa sanguínea fija media cambió ligeramente (pero con importancia estadística) durante la terapia de ambos grupos. Sin embargo, este cambio fué bastante moderado y 3 meses después de terminada la terapia la glucosa sanguínea fue bastante normal. La desaparición de la constante de la glucosa en el plasma durante la GTT itnravenosa no tuvo variación en los dos grupos durante los 3 meses de terapia contraceptiva oral. Ninguna mujer, de ninguno de los dos grupos, necesitó insulina durante la terapia.

Los estudios adicionales sobre los efectos a largo plazo sobre el metabolismo de carbohidratos, así como sobre el uso de diferentes tablas de dosificación, se hacen necesarios para evaluar la seguridad del diacetato etinodiol. 